\title{
The Case for In-Memory Inferencing of Quantized CNNs at the Edge
}

This paper was downloaded from TechRxiv (https://www.techrxiv.org).

\section{LICENSE}

CC BY 4.0

SUBMISSION DATE / POSTED DATE

28-02-2022 / 07-03-2022

CITATION

Falcao, Gabriel; Ferreira, João Dinis (2022): The Case for In-Memory Inferencing of Quantized CNNs at the Edge. TechRxiv. Preprint. https://doi.org/10.36227/techrxiv.19249511.v1

$\mathrm{DOI}$

10.36227/techrxiv.19249511.v1 


\title{
The Case for In-Memory Inferencing of Quantized CNNs at the Edge
}

\author{
Gabriel Falcao \\ Department of Electrical and Computer Engineering of the Faculty of Science and Technology of the \\ University of Coimbra, and Instituto de Telecomunicações, Portugal \\ João Dinis Ferreira \\ Department of Electrical Engineering and Information Technology, ETH Zürich and SAFARI Research \\ Group, Switzerland
}

\begin{abstract}
As artificial intelligence becomes a pervasive tool for the billions of loT devices at the edge, the data movement bottleneck imposes severe limitations on these systems' performance and autonomy. Processing-in-Memory emerges as a way to mitigate the data movement bottleneck while satisfying the stringent performance, energy efficiency, and accuracy requirements of edge imaging applications that rely on convolutional neural networks.
\end{abstract}

\section{INTRODUCTION}

The globalization of affordable Internet access has spurred a revolution in computer architectures, characterized by the accelerated widespread adoption of smartphones, tablets, and other smart devices, which are now commonplace [1]. The rise of Internet of Things (IoT) applications in a wide range of domains (e.g., personal computing, education, industry, military, healthcare, digital agriculture) brings with it the ability to integrate billions of devices on the Internet, as depicted in Figure 1. This integration presents unprecedented challenges, such as the need for inexpensive computation and communication, capable of crunching the increasing volumes of data generated every day.

As Al becomes a pervasive tool for billions of loT devices at the edge, the data movement bottleneck imposes limitations on these systems' performance and autonomy.
The IoT paradigm also promises a more intimate connection between the cyber and physical worlds, as data becomes a ubiquitous asset exchanged between all manner of connected (smart) devices. Moreover, the data flow is often bidirectional- taking place not just from the physical to the cyber world, but also from the cyber to (what is possible in) the physical world.

Bringing this vision to fruition will require that IoT devices exhibit AI. The most promising approaches today are based on empowering systems with the ability to learn autonomously from experience by assimilating large amounts of data-using so-called machine learning (ML) algorithms-with a particular focus in deep learning and image inference.

\section{Al Demands at the Edge Will Grow}

The total volume of digital data created, replicated and consumed within a year has surpassed dozens of ZB ( $10^{21}$ Bytes) in 2020 and the International Data Corporation estimates that this number will grow to hundreds of $\mathrm{ZB}$ in coming 


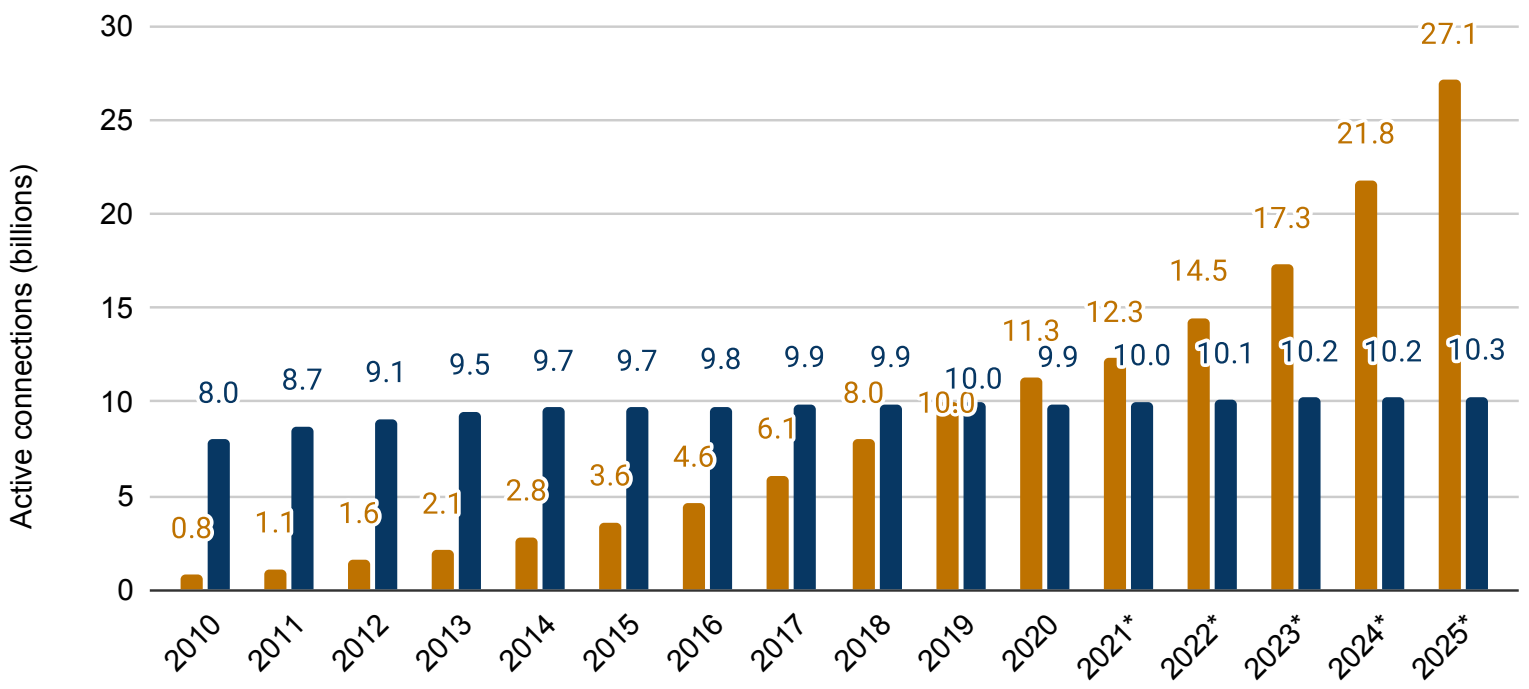

Figure 1: Number of worldwide IoT vs. non-IoT active device connections on the Internet from 2010 to 2025. Data extracted from https://iot-analytics.com/number-connected-iot-devices/. * Projected values.

years [2]. The COVID-19 pandemic contributed to this figure due to widespread work-fromhome mandates and a sudden increase in video conferencing and streaming data. A significant portion of this data is consumed at the edge, with processing many times performed entirely on smartphones and embedded systems. The rise of many other AI-based applications applied to the big data revolution exacerbates this problem by placing increasing stress on computing and memory systems, in particular those operating at the edge.

\section{Why Process Al at the Edge?}

We propose that the case for edge computing as the enabler of sustainable AI scaling is strengthened by the observation that a sizable portion of the data generated by modern digital systems originates from sensors located at the edge-under this paradigm, data is processed where it is generated, as Figure 2 illustrates. Furthermore, with the growth in data generation and the expansion of demand for AI applications at increasing rates, at least five goals justify moving the processing of AI to the edge [3], [4], [5]:

- Latency: Many applications have an interactive nature and thus cannot endure long latencies, especially for memory, storage, and network requests.

- Reliability: Communication networks are not reliable at all places, at all times. To ensure maximum uptime, AI-based decision-making must not rely on always-available communication networks.

- Privacy/security: Some applications require sensitive data to be kept in a controlled local environment, avoiding its circulation to/from the cloud. Examples are medical, finance, or autonomous driving among many others.

- Bandwidth: Data that is processed near where it is collected does not need to be sent to the cloud, which reduces the overall bandwidth demand on the network and the edge computing systems.

- Data provenance: Provenance issues may prevent data from being processed far from where it is generated. Data center storage may need to comply with regional data protection legislation such as General Data Protection Regulation (GDPR) in Europe and Personal Information Protection Act (PIPA) in Canada.

To achieve these goals, computer architects must adopt a holistic vision of the combined cloud+edge system to keep the unnecessary movement of data between components to a minimum by processing data where it is generated and stored, as this is the dominant performance and energy bottleneck [6], [7]. 


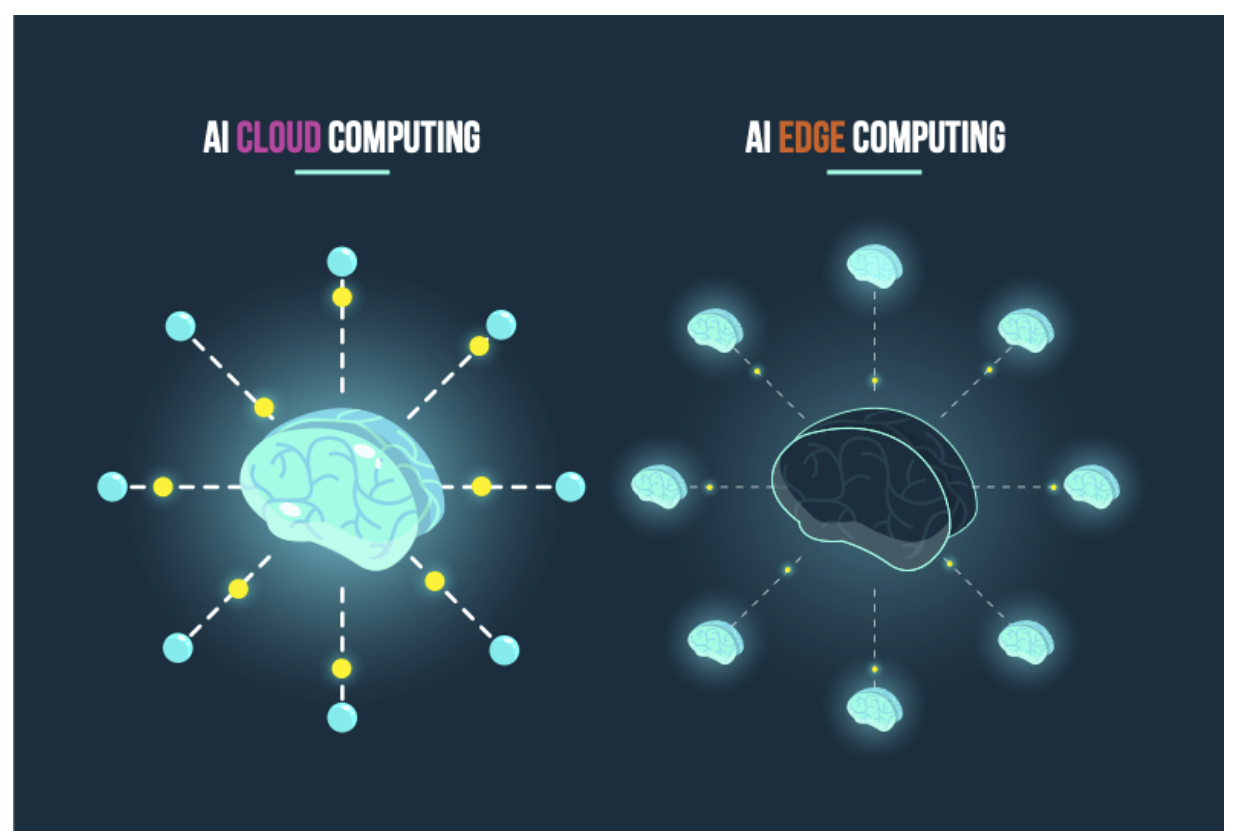

Figure 2: The migration of AI to the edge: in contrast with the conventional approach (left), with the emergence of cloud-edge hierarchies, AI moves to the edge layer (right). This new paradigm creates pressure on the processing nodes at the edge, but it also decreases the time and energy spent performing communications with the cloud, while introducing benefits in data privacy, latency and others.

Five goals justify moving the processing of Al to the edge: latency, reliability, privacy/security, bandwidth and data provenance.

\section{Al AT THE EDGE: DATA \\ CHALLENGES, ML SOLUTIONS}

At present, neural networks are widely used in many domains and they are also becoming integral components of other emerging applications, such as self-driving cars, always-on biosignal monitoring, augmented and virtual reality, critical IoT, and voice communications (which represent up to $25 \%$ of the use cases of $5 \mathrm{G}$ at the edge), all of which require AI algorithms to operate on high volumes of data at the edge (see Figure 3). The systems that support these emerging applications will be expected to make decisions faster-and, often, better-than their human counterparts, with support for continuous fine-tuning of their decision-making process by factoring in ever-increasing volumes of data for training and inference.

In particular, convolutional neural networks (CNNs) are the de facto standard for image- based decision-making tasks. These models heavily utilize the convolution and the multiply-andaccumulate (MAC) operations, which represent more than $90 \%$ of the total cost of computation [8]. For this reason, state-of-the-art neural network accelerators (e.g., Google's Tensor Processing Unit) have focused on optimizing the performance and energy efficiency of the MAC operation.

\section{Characterizing and Optimizing CNN \\ Architectures}

Typical CNNs comprise hundreds of millions of parameters and often require the execution of billions of operations per second to achieve realtime performance. As their accuracy improves, CNNs include more parameters and layers, becoming wider and deeper. The use of compact data representations provided by quantization mitigates some of the overheads of these more complex network architectures and enables high degrees of parallelism and data reuse, which are especially useful in constrained processing environments. Data quantization significantly reduces the computation and storage requirements of neural networks by decreasing the bit width of the model's weights. However, quantizing these 


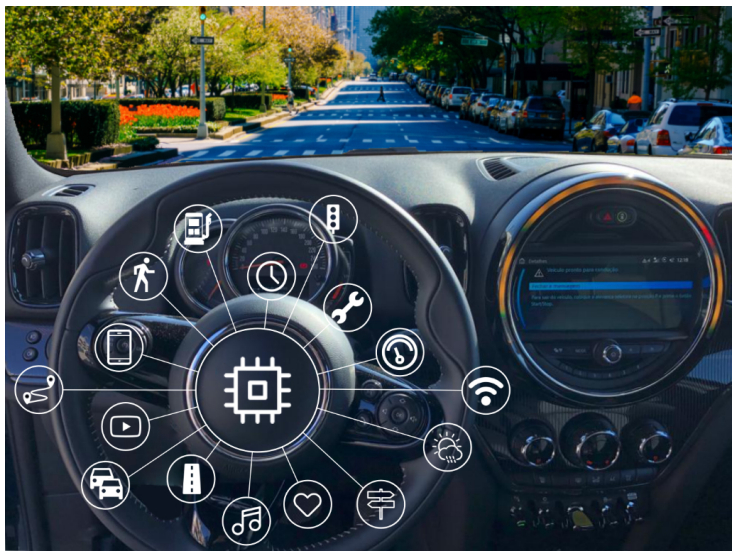

(a)

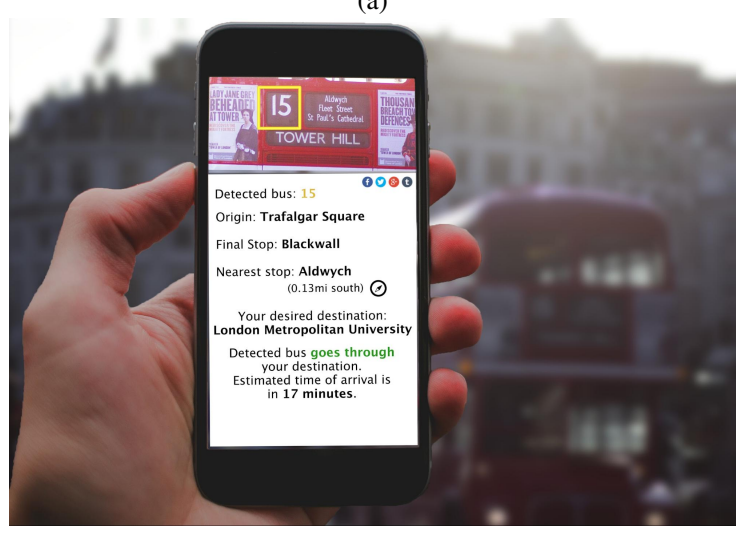

(c)

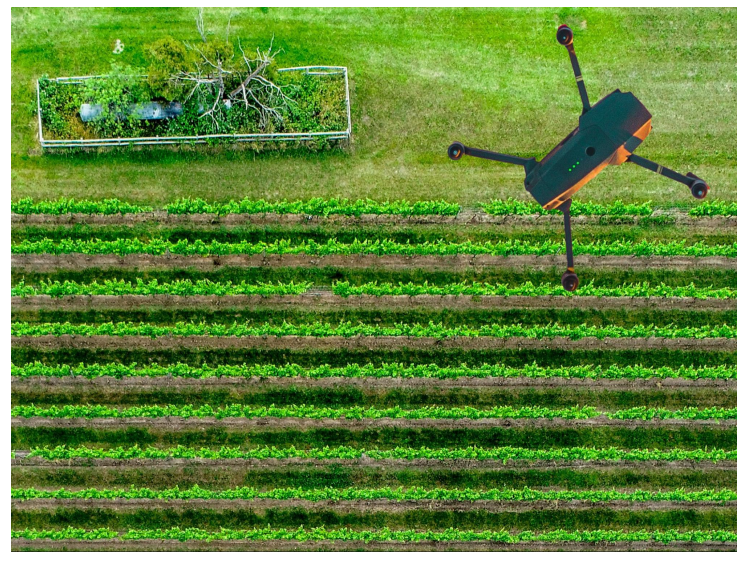

(b)

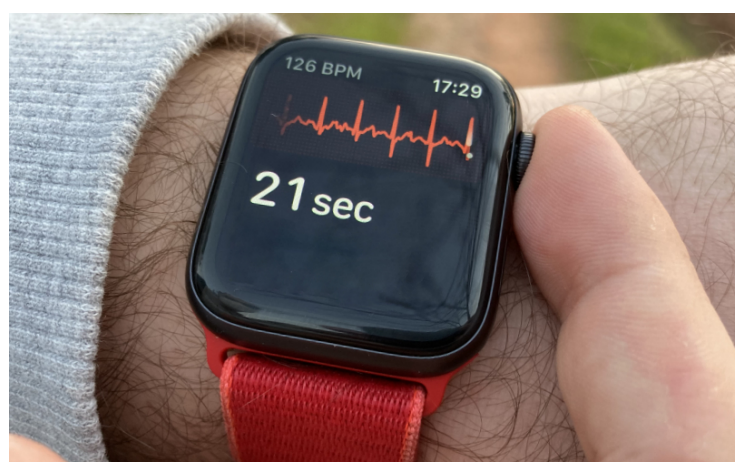

(d)

Figure 3: Examples of a) autonomous vehicles, b) digital agriculture, c) smartphones, and d) smart IoT devices that process substantial volumes of data while running AI kernels at the edge. Attribution: Unsplash ${ }^{1,2}$.

values to under 8 bits while retaining accuracy requires manual effort, the tuning of hyperparameters, and intensive re-training.

While the training process requires a high dynamic range, inference does not: in most cases, 2- to 4-bit precision achieves the desired levels of accuracy [9]. Going further, it is even possible to approximate CNNs by binarizing (i.e., quantizing to 1-bit) their input, weights and/or activations [9], [10]. In many practical settings, and in particular for edge computing, the performance and energy efficiency benefits of binary neural networks outweigh the accuracy loss. A further benefit afforded by binary neural networks is the ability to approximate the convolution operation required by CNNs, by combining the much more efficient bitwise XNOR and bit counting operations.

\footnotetext{
${ }^{1}$ https://unsplash.com/photos/0x065I5N6Vw

${ }^{2}$ https://unsplash.com/photos/lsqLJCvrOpc
}

Table 1 illustrates the memory and processing requirements of five widely used $\mathrm{CNN}$ models across a range of devices, from data center servers to IoT nodes. These were selected from a large set of neural network algorithms to fit the edge nodes, namely in terms of memory required to run the models. We show their size as a function of weight/activation bit widths (32-bit, 2-bit, 1bit), as well as the number of MAC operations used by each network.

\section{KEY TAKEAWAY 1}

Quantized data structures minimize data movement and storage requirements, facilitating the execution of $\mathrm{Al}$ kernels at the edge. 


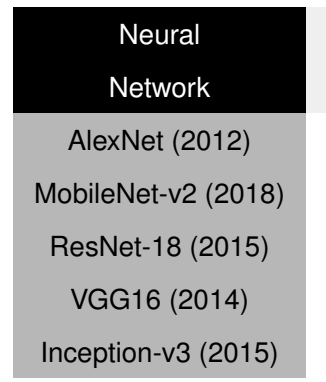

$\begin{array}{ccc}\text { Size 32/32 } & \text { Size 2/2 } & \text { Size 1/1 } \\ \text { (W/A) (MB) } & \text { (W/A) (MB) } & \text { (W/A) (MB) } \\ 237 & 14.8 & 7.4 \\ 13 & 0.8 & 0.4 \\ 45 & 2.8 & 1.4 \\ 515 & 32.2 & 16.1 \\ 91 & 5.6 & 2.8\end{array}$

$\begin{array}{cc}\text { \#Params. } & \text { \#MAC operations } \\ (\mathrm{M}) & (\mathrm{G}) \\ 62 & 0.7 \\ 3.4 & 0.3 \\ 12 & 1.8 \\ 135 & 15.5 \\ 24 & 2.8\end{array}$

Table 1: A collection of five popular convolutional neural networks, all using the ImageNet dataset. The table indicates the number of parameters, and multiply and accumulate operations for all the networks (these operations represent more than $90 \%$ of total computation). W/A refers to the bit widths of the Weights and the Activations for each level of quantization. All networks have 224x224 input resolution.

\section{Specialization as the Enabler of High-Performance Al}

Modern deep learning algorithms demand substantial computational, memory, and energy requirements [11], which makes their implementation on edge devices challenging. However, this challenge can be addressed by exploiting two unique characteristics of ML algorithms: first, each class of deep learning algorithm relies on a limited set of specialized operations; second, in many cases, these algorithms provide good accuracy even when they make use of low-bitwidth operations [12].

In recent years, several frameworks (e.g., Tensorflow, PyTorch, TensorRT) helped bridge the semantic gap between the high-level description of a neural network model and its underlying hardware mapping using specialized instructions. This is achieved by performing operations in a bulk parallel manner, minimizing memory accesses and maximizing compute resource utilization.

\section{KEY TAKEAWAY 2}

Specialized arithmetic and logic instructions for Al show potential to accelerate the performance and energy efficiency of edge processors.

\section{EFFICIENT EDGE AI: ARCHITECTING DATA-CENTRIC SYSTEMS}

The observations and constraints introduced above lead to the formulation of a set of welldefined target metrics for $\mathrm{AI}$ at the edge:

- Accuracy: the success rate of the AI task [9] (e.g., image classification, object detection, sentence generation, translation).

- Throughput: the rate of processing of input data; many real-time AI applications that support video must sustain processing rates in the order of thousands of frames per second (FPS) (e.g., 2300 FPS in Tesla self-driving cars [13]; or hundreds to thousands of FPS in ultrasound medical devices).

- Latency: the critical path delay associated with the processing of a single input element; $5 \mathrm{G}$ standards define a maximum latency of $1 \mathrm{~ms}$ for positioning and tracking systems; selfdriving cars must provide latencies in the same order of magnitude [13].

- Power and energy: most edge devices are battery-powered, and maximizing battery life is a key design target. For reference, the operation of self-driving cars requires a power supply of up to $2.5 \mathrm{~kW}$ [13].

- Data precision: AI data need not always be represented in 64- or 32-bit floating-point precision. For many inference applications, integer precision of 8 bits or less suffices [14].

\section{The Good Old Processor-Centric Computing Model}

The late 20th and early 21 st centuries saw the widespread use of the processor-centric computational model. In this model, programs and data are stored in memory and processing takes place in specialized Arithmetic and Logic Units (ALUs). Together with Moore's law, the introduction of caches, branch predictors, out-oforder execution, multithreading, and several other hardware and software optimizations enabled a 
steady and mostly unfazed series of performance improvements over the last decades.

In contrast, memory systems have improved at a much slower pace. This performance gap between the processor and the main memory-supported by the fact that these technologies are still several process node generations apart-has given rise to a critical data movement bottleneck, dubbed the memory wall [15]. Memory is the dominant performance and energy bottleneck in modern computing systems: data movement is much more expensive than computation, both in latency and energy. The data movement bottleneck will remain relevant as the number of smart devices connected to the Internet-as of this writing, already in the billions, as depicted in Figure 1-continues to grow.

\section{KEY TAKEAWAY 3}

The mitigation of unnecessary data movement minimizes the latency and energy requirements for the processing of $\mathrm{Al}$, in particular for energyconstrained edge nodes.

\section{A Compelling Possibility: Processing Data Where it Resides}

As the demand for inferencing at the edge grows, accessing data more efficiently becomes increasingly relevant. The proposed improvements span 1) data reuse by exploiting temporal and physical locality, 2) algorithm design, with the introduction of optimized neural network topologies and the use of quantization, 3) specialized hardware, introducing dedicated vectorized instructions designed to address the demands of these workloads (e.g., SIMD MAC operations), and 4) Processing-in-Memory (PiM) architectures. When performed at the edge, PiM enables higher throughput for AI applications, without compromising device autonomy [10].

PiM solutions differ primarily in their proximity to the data [15]: in the Processing-nearMemory (PnM) paradigm, computation takes place close to where data resides, but in a different medium-for example, in the logic layer of a 3D-stacked memory; in contrast, the Processingusing-Memory (PuM) paradigm takes advantage of the storage medium's physical properties to perform computation (e.g., ReRAM or DRAM memory cells [16]).

Data movement is much more expensive than computation, both in latency and energy.

\section{Processing-near-Memory 3D-stacked}

memories are an emerging type of memory architecture that enables the vertical stacking of memory layers on top of a logic layer. This logic layer can be designed to feature hardware support for several operations, thus enabling computation inside the memory units [17].

Processing-using-Memory DRAM technology is especially well-suited to support bitwise operations since adjacent memory rows can communicate with one another through their bitlines.

Ambit [18] supports bulk bitwise majority/AND/OR/NOT functions by exploiting the analog operation of DRAM. The combination of these operations enables the design of full applications. Recent studies show that Ambit's core operating principle can be performed in commodity off-the-shelf DRAM chips with no changes to DRAM [19]. Ambit improves performance by $30-45 \times$ and reduces energy consumption by 25 $60 \times$ for the execution of bulk bitwise operations, resulting in $4-12 \times$ overall speedup in database queries.

SIMDRAM [20] creates an optimized graph representation of a user-defined arbitrary operation using bitwise majority and NOT operations, which can be performed using the triple-row activation command defined in Ambit. Finally, the SIMDRAM control unit orchestrates the computation from start to finish by executing the previously defined DRAM commands. SIMDRAM improves performance by $88 \times / 5.8 \times$ and reduces energy consumption by $257 \times / 31 \times$ compared to CPU/GPU execution.

pLUTo [21] extends the flexibility and performance of PuM by introducing a mechanism for bulk in-DRAM value lookups. The lookups take place entirely within the DRAM subarray, and therefore do not require that data be moved off-chip at any point. Using pLUTo, it is possible 
to implement arbitrarily complex functions as table lookups (so long as the memory arrays are sufficiently large to accomodate them) while minimizing the overall movement of data. pLUTo improves performance by $33 \times / 8 \times$ and reduces energy consumption by $110 \times / 80 \times$ compared to CPU/GPU execution.

PRIME [22] and ISAAC [23] are two promising neural network accelerators based on resistive random access memory (ReRAM). These proposals leverage the ReRAM crossbar array to perform matrix-vector multiplication efficiently in the analog domain. These solutions report performance and energy consumption improvements of up to $2360 \times$ and $895 \times$, respectively, relative to state-of-the-art neural processing unit designs.

\section{Enabling the Adoption of PiM}

The fact that quantized neural networks rely on simple operations makes them a prime target for exploiting the use of PiM to process AI kernels at the edge. Performing more straightforward computation close to where data resides greatly reduces the overall movement of data, which improves latency, throughput, and energy efficiency. We propose that these can be applied even more efficiently to quantized and binary convolutional neural networks, which use XNOR and bit count operators.

To validate the performance and energy merits of PiM, in the next section we perform a quantitative analysis of the improvements to $\mathrm{CNN}$ image inference at the edge, offered by Ambit, a DRAM-based PuM architecture that supports bitwise logic operations.

\section{TO PIM OR NOT TO PIM: A QUANTITATIVE ANALYSIS}

This section evaluates the accuracy, performance, and energy costs of performing inference for binary and quantized versions of the most recent neural networks introduced in Table 1. To this end, Table 2 shows the energy consumption of Ambit-based PiM designs to perform inference on each of these networks. These estimates account for the cost of performing the dominant MAC operations required by the convolutions. We show that different accuracy-performance-energy trade-offs can be obtained. In Table 3, we analyze the performance of the four most recent neural networks from Table 1, targeting 15 and 60 FPS, for 3-, 2- and 1-bit precision. We empirically observe that these quantized models ensure adequate accuracy for many applications. We observe that ingenious quantization techniques enable either 1) further compression of even the smallest neural network models or 2) the portability of very large networks for use in power-constrained devices, with small, tunable, accuracy losses.

Accuracy. The average accuracy loss is $6.8 \%$, and in the best case the loss is as low as $2.2 \%$. In cases where the accuracy of the 1-bit network may result in too great of an accuracy loss, we observe that settling for 2- or 3-bit quantization often yields good accuracy values.

Performance. The described Ambit-based accelerator achieves 15 FPS in 8 cases out of 12 , and 60 FPS for 4 . In contrast, the Qualcomm Snapdragon, Intel Xeon and Edge TPU baselines sustain 15 FPS in, respectively, 0, 3, and 9 cases out of 12 , and 60 FPS in 0,0 , and 9 cases out of 12. The average speedup of the Ambitbased accelerator over each of these baselines is $58.8 \times, 21.3 \times$, and $4.6 \times$. We draw two key conclusions. First, we observe that the Edge TPU can only sustain a processing rate of 3.4 FPS for VGG-16, due to this network's high number of parameters. This illustrates the poor scalability of current specialized neural network accelerators to process very large networks. Second, the flexible degree of parallelism in the PiM implementation, attained through the operation of multiple subarrays in parallel, allows the inference time to scale quasilinearly with the size of the network, which enables a near-constant inference time in the largest networks.

Energy. We observe average energy savings of $35.4 \times$ for 1-bit, relative to 32-bit precision. Energy gains are linearly proportional to the degree of quantization.

\section{OUTSTANDING CHALLENGES}

As the volume of data to process approaches our installed processing capacity [24], the successful implementation of $\mathrm{AI}$ at the edge will depend on the development of optimized architectures that are able to perform AI tasks while meeting strict performance and energy efficiency requirements. After the introduction of thousandcore processors and the expansion of levels of 


\begin{tabular}{ccccccccc} 
& \multicolumn{3}{c}{ Baseline Accuracy (\%) } & \multicolumn{3}{c}{ Energy of PiM Designs (mJ) } \\
\cline { 2 - 8 } Neural Network & Top-1\% & Top-1\% & Top-1\% & Baseline $\diamond$ & PiM & PiM & PiM* \\
& $32 / 32(\mathrm{~W} / \mathrm{A})$ & $2 \leq \mathrm{W} / \mathrm{A} \leq 8$ & $1 / 1(\mathrm{~W} / \mathrm{A})$ & $32 / 32$ & $3 / 3$ & $2 / 2$ & $1 / 1$ \\
AlexNet & 60.0 & 58.2 & 57.0 & 344 & 173 & 91 & 10 \\
MobileNet-v2 & 72.0 & - & 54.4 & 91 & 46 & 24 & 3 \\
ResNet-18 & 69.1 & $67.0 \dagger$ & 64.8 & 1090 & 548 & 288 & 31 \\
VGG16 & 71.1 & 69.1 & 68.9 & 9390 & 4716 & 2483 & 265 \\
Inception-v3 & 78.0 & $76.4 \ddagger$ & - & 3450 & 1734 & 913 & 97
\end{tabular}

Table 2: Energy consumed per inference for the five neural networks represented in Table 1, for the ImageNet dataset. Energy is calculated for execution on both ARM CPUs at the edge (baseline) and also using an analytical model, for $\mathrm{PiM}$ technology. For full access to the original references, please see https://github.com/joaodinissf/qcnn-accuracy/. $\diamond$ Qualcomm Snapdragon 865 with 4 ARM Cortex-A77 and 4 A55 CPUs using multilevel cache and DRAM-based main memory. Although the TDP for this processor is $5 \mathrm{~W}$, we assume a more realistic power draw of $3.06 \mathrm{~W}$, as derived by averaging its power consumption during the execution of the SPEC2006fp benchmark. Emulating the ARM baseline does not show competitive performance for 1-, 2-, or 3-bit precision, as it would require higher energy consumption than the 32/32-bit baseline.

* Assumes 1-bit quantization of the neural network's weights and activations and that all operations are performed in-memory on an LPDDR4 with clock frequency $2133 \mathrm{MHz}$ and $\mathrm{tRCD}=\mathrm{tCL}=\mathrm{tRP}=15 \mathrm{~ns}$.

$\dagger$ Employs ternary data representation for both weight and activation values.

$\ddagger$ Employs 4-bit weights and 8-bit activations. The weights of the first and last layers are 8-bit values.

parallelism in the compute units, computer architects must now turn to the memory to determine its role in the design of next-generation highperformance and high-efficiency systems. While PiM architectures have shown promise [25], enabling their adoption at the edge layer will require answers to the following open questions:

\section{For Manufacturers: Designing PiM}

- Software/Hardware Co-Design: The integration of PiM technology and compiler with new firmware and the operating system should be facilitated to enable the design of edge systems with maximal performance and energy efficiency in order to be able to meet the computational demands of AI applications and use cases. PiM can be specialized for the edge by supporting common image processing operations, which are especially relevant for performance.

- Low Design Complexity and Cost: PiM designs should be made sufficiently low-cost to entice hardware manufacturers to integrate them into their products. This will entail the development of early-access commercial prototypes and proof-of-concept products that encourage early adopters to disseminate the technology and offset R\&D costs. PiM technology is reaching a point of maturity, and it will soon make its way to self-driving cars, healthcare, digital agriculture, and other edge AI applications with massive total addressable markets.

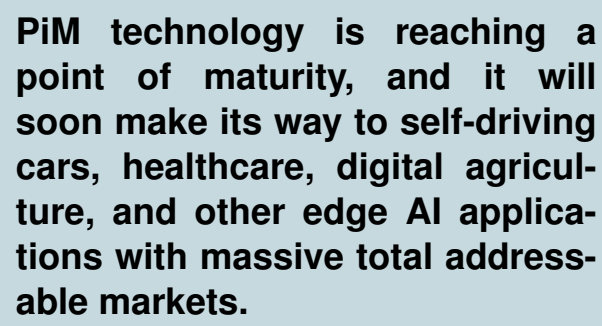

- Multi-Tiered PiM Architectures: As PiM technology matures, it will populate multiple levels of the existing memory hierarchy with complementary processing components, each with their own benefits and drawbacks. For example, Processing-in-Cache architectures have been proposed, which trade off the capacity of DRAM for greater speed and support for a wider range of operations. Analogously, Processing-in-Storage (typically oriented towards non-volatile memories) is well-suited for the execution of simpler operations on vast volumes of data, with very high throughput.

- The Compiler: PiM substrates will require custom compilers; these compilers can be a tool to aid programmers in the identification of common memory access and computational 


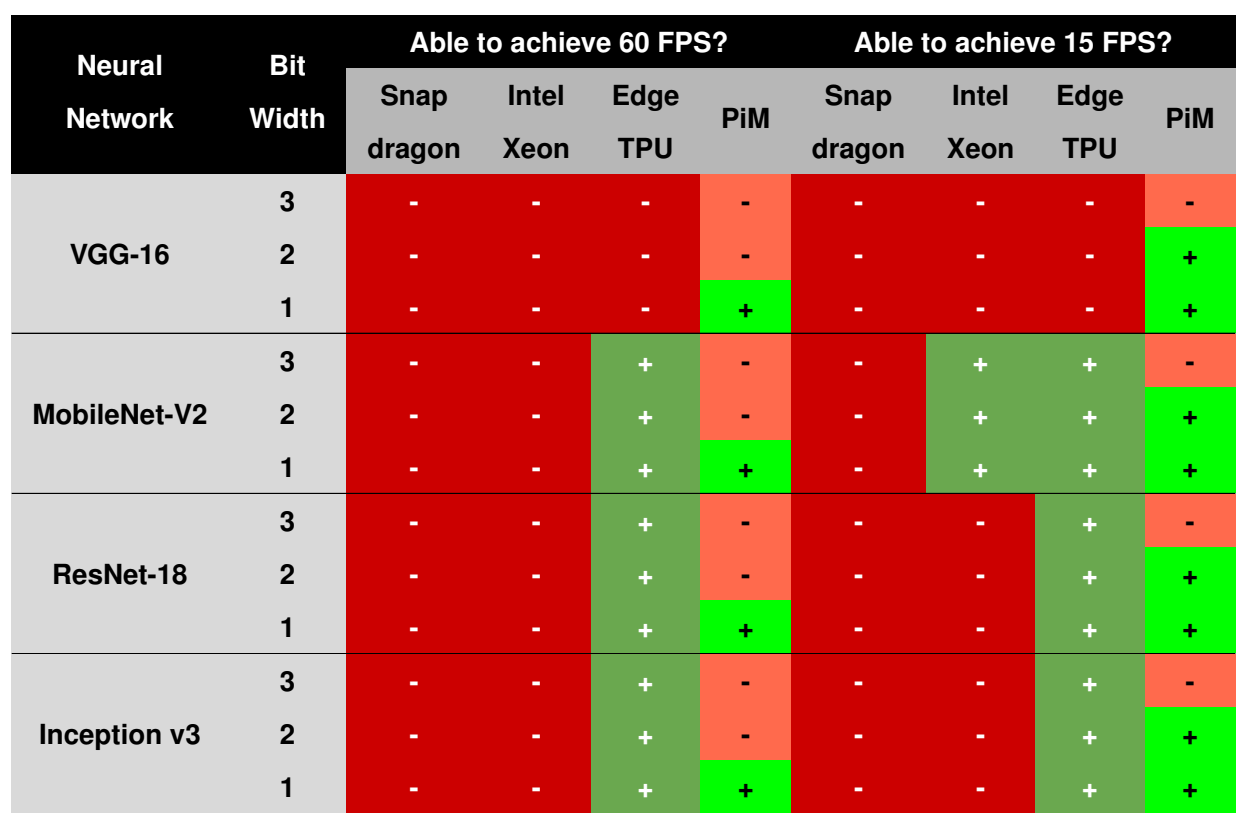

Table 3: Supported inference throughput (in FPS) for Qualcomm Snapdragon 865, Intel Xeon Gold 6154 and Edge TPU baselines, compared with an Ambit-based PiM architecture. Red cells indicate lack of real-time support, green cells indicate real-time support

patterns, in order to automate certain steps of the compilation process to yield maximal performance (e.g., the adoption of ideal data mapping, or the circumvention of bottlenecking memory accesses).

\section{For End-Users: Developing for PiM}

- Algorithm Design: Mapping existing and emerging applications to PiM substrates requires the algorithm to exploit data quantization, the exposed parallelism, and the coalescing of memory accesses to offset the highcost operations in the system, which are especially costly at the edge. The programmer is responsible for achieving the desired trade-off between performance, energy efficiency and accuracy to satisfy the requirements of their target AI application.

- The Development Framework: A low-cost approach to empower the compiler with knowledge about the application's dataflow is to create an intuitive and expressive API that abstracts as many of the high-efficiency operations supported by the PiM substrate in an easy-to-use way. It is tempting to draw an analogy between the current state of PiM and the early days of GPGPU computing. CUDA and OpenCL were instrumental for enabling the mass-scale adoption of GPUs, and a similar API must assume that role for PiM.

\section{KEY TAKEAWAY 4}

The creation of a PiM development framework will greatly facilitate adoption and development for PiM's early adopters. This especially eases the learning curve for programmers of edge and consumerfacing applications and contributes to PiM's widespread adoption.

- Benchmarking Tools: Standard benchmarking, profiling, simulation and analysis tools enable the comparison of different architectures, and are therefore essential for the research and development stage of emerging PiM. This is especially important for emerging applications, as is the case of many edge AI workloads. Similarly to the role that MLPerf (the result of a collaboration of a consortium of AI leaders from academia, research labs, and industry) plays in the development of machine learning algorithms, a similar set of standardized tests would enable the development of PiM to remain steadfast and open. 


\section{WHAT THE FUTURE WILL BRING AND THE ROLE OF PIM}

Demand for AI at the edge will continue to grow. We propose that high-performance and energy-efficient PiM-based edge architectures for the processing of AI should:

1) Support quantized data structures. As shown exhaustively in the literature, particularly for inference, the use of reducedbit-width representations yields only small losses in accuracy. This is particularly useful for reducing the movement of data within/from/to the memory subsystem and for increasing the degree of vectorization and parallelism.

2) Support specialized instructions. Bitwise and LUT-based operations can be implemented in the memory substrate as rowlevel operations or in the logic layer, with sizable benefits for running AI kernels with low hardware complexity, high bandwidth, and high parallelism.

3) Avoid the unnecessary movement of data. Support for a compiler that detects what part of the AI kernel should run on the processor and what part should run in memory. Not only are different portions of the kernel more suitable for distinct subsystems, but it is also fundamental to balance the workload among them to maximize performance. The minimization of energy consumption is another target that the computer architecture community should aim for.

4) Foster a PiM ecosystem. Achieving critical mass in the adoption of PiM is a crucial milestone for its success, and reaching it requires a programmer-friendly interface, intuitive compilers, and comprehensive test-suites, with a set of industrystandard benchmarks.

\section{Where Do We Go From Here?}

Several PiM architectures have demonstrated the ability to perform AI tasks with unprecedented levels of efficiency [18], [19] by meeting the takeaways presented above. However, existing PiM designs support a limited range of operations, and further work is necessary to meet the requirements of $\mathrm{AI}$ tasks.
Smart devices may soon come to incorporate both conventional and PiM-enabled memories.

PiM is not a silver bullet, and it will not supersede conventional computing —although we may soon find that computers and other smart devices benefit from incorporating both conventional processing units and PiM-enabled memories. When designing new architectures, computer architects must remain mindful that data movement is expensive in both latency and energy. Emerging technologies and architectures enable the mitigation of data movement and, as such, pave a path for the design of more efficient computing devices. The current paradigm will only take us so far; PiM presents a compelling alternative.

\section{ACKNOWLEDGMENTS}

This work was partially supported by Instituto de Telecomunicações and Fundação para a Ciência e a Tecnologia, Portugal, under grants EXPL/EEI-HAC/1511/2021, PTDC/EEIHAC/30485/2017 and UIDB/EEA/50008/2020.

\section{REFERENCES}

1. P. Pandey and D. Pompili, "Handling limited resources in mobile computing via closed-loop approximate computations," IEEE Pervasive Computing, vol. 18, no. 1, pp. 39-48, 2019.

2. I. D. Corporation, The Digitization of the World - From Edge to Core". IDC White Paper, 2018.

3. M. Satyanarayanan, "Edge Computing: A New Disruptive Force", Keynote at SYSTOR," 2020.

4. H. Fuketa and K. Uchiyama, "Edge Artificial Intelligence Chips for the Cyberphysical Systems Era," Computer, 2021.

5. M. Campbell, "Smart Edge: The Effects of Shifting the Center of Data Gravity Out of the Cloud," Computer, 2019, conference Name: Computer.

6. A. Boroumand, S. Ghose, Y. Kim, R. Ausavarungnirun, E. Shiu, R. Thakur, D. Kim, A. Kuusela, A. Knies, and P. Ranganathan, "Google workloads for consumer devices: Mitigating data movement bottlenecks," ASPLOS, 2018.

7. J. L. Hennessy and D. A. Patterson, "A new golden age for computer architecture," CACM, 2019. 
8. N. Jouppi, C. Young, N. Patil, D. Patterson, G. Agrawal, R. Bajwa, S. Bates, S. Bhatia, N. Boden, and A. Borchers, "In-datacenter performance analysis of a tensor processing unit," in ISCA, 2017.

9. I. Hubara, M. Courbariaux, D. Soudry, R. El-Yaniv, and Y. Bengio, "Quantized neural networks: Training neural networks with low precision weights and activations," The Journal of Machine Learning Research, 2017.

10. M. Rastegari, V. Ordonez, J. Redmon, and A. Farhadi, "Enabling $\mathrm{Al}$ at the edge with XNOR-networks," Communications of the ACM, 2020.

11. V. Sze, Y.-H. Chen, T.-J. Yang, and J. Emer, "Efficient processing of deep neural networks: A tutorial and survey," in IEEE, 2017.

12. J. Marques, J. Andrade, and G. Falcao, "Unreliable memory operation on a convolutional neural network processor," in 2017 IEEE (SiPS, 2017.

13. E. Talpes, D. Sarma, G. Venkataramanan, P. Bannon, B. McGee, B. Floering, A. Jalote, C. Hsiong, S. Arora, and A. Gorti, "Compute solution for Tesla's full selfdriving computer," IEEE Micro, 2020.

14. P. Duarte, P. Tomas, and G. Falcao, "SCRATCH: an endto-end application-aware soft-GPGPU architecture and trimming tool," in MICRO, 2017.

15. O. Mutlu, S. Ghose, J. Gómez-Luna, and R. Ausavarungnirun, "A Modern Primer on Processing in Memory," 2020.

16. M. Soeken, P.-E. Gaillardon, S. Shirinzadeh, R. Drechsler, and G. De Micheli, "A PLiM Computer for the Internet of Things," Computer, 2017.

17. P. Rosenfeld, "Performance exploration of the hybrid memory cube," 2014, place: Diss.

18. V. Seshadri, D. Lee, T. Mullins, H. Hassan, A. Boroumand, J. Kim, M. Kozuch, O. Mutlu, P. Gibbons, and T. Mowry, "Ambit: In-memory accelerator for bulk bitwise operations using commodity DRAM technology," in MICRO, 2017.

19. F. Gao, G. Tziantzioulis, and D. Wentzlaff, "ComputeDRAM: In-memory compute using off-the-shelf DRAMs," in MICRO, 2019.

20. N. Hajinazar, G. Oliveira, S. Gregorio, J. Ferreira N. Ghiasi, M. Patel, M. Alser, S. Ghose, J. Gómez-Luna, and O. Mutlu, "SIMDRAM: a framework for bit-serial SIMD processing using DRAM," in ASPLOS, 2021.

21. J. D. Ferreira, G. Falcao, J. Gómez-Luna, M. Alser, L. Orosa, M. Sadrosadati, J. S. Kim, G. F. Oliveira, T. Shahroodi, A. Nori, and O. Mutlu, "pLUTo: In-DRAM Lookup Tables to Enable Massively Parallel GeneralPurpose Computation," arXiv:2104.07699 [cs], 2021, arXiv: 2104.07699.
22. P. Chi, S. Li, C. Xu, T. Zhang, J. Zhao, Y. Liu, Y. Wang, and Y. Xie, "PRIME: A Novel Processing-in-Memory Architecture for Neural Network Computation in ReRAMBased Main Memory," ACM SIGARCH Computer Architecture News, 2016.

23. A. Shafiee, A. Nag, N. Muralimanohar, R. Balasubramonian, J. Strachan, M. Hu, R. Williams, and V. Srikumar, "ISAAC: A convolutional neural network accelerator with in-situ analog arithmetic in crossbars," ACM SIGARCH Computer Architecture News, 2016.

24. M. Duranton, K. De Bosschere, B. Coppens, C. Gamrat, T. Hoberg, H. Munk, C. Roderick, T. Vardanega, and O. Zendra, "The HiPEAC Vision 2021," HiPEAC CSA, 2021.

25. P. Amato, P. Radojković, P. Carpenter, P. Esmaili-Dokht, R. Cimadomo, H.-P. Charles, A. Sebastian, and P. Amato, "Processing in Memory: The Tipping Point," 2021.

Gabriel Falcao received his $\mathrm{PhD}$ degree from the University of Coimbra, Portugal, in 2010. He is a Researcher at Instituto de Telecomunicações and a Tenured Assistant Professor at the Department of Electrical and Computer Engineering of the University of Coimbra. He was a Visiting Professor at EPFL in 2011/12 and again in 2017, and a Visiting Academic at ETH Zürich in 2018. Gabriel is co-author of more than 100 peer-reviewed publications and his research interests include parallel computer architectures for data-intensive signal processing applications. $\mathrm{He}$ is currently working on building low-power architectures for $\mathrm{Al}$, with a particular focus on processingin-memory hardware. In 2020 Gabriel Falcao was General Co-Chair of the IEEE SiPS, whose theme was "Signal Processing at the Edge". Gabriel is a Senior Member of IEEE and a Member of the HiPEAC Network of Excellence in Europe. Contact him at gabriel.falcao@uc.pt.

João Dinis Ferreira holds a Bachelor's Degree in Electrical and Computer Engineering from the University of Coimbra, where he graduated in the top $3 \%$ of his class. He is currently a Research and Teaching Assistant at ETH Zürich, where he is pursuing a Master's Degree in Electrical Engineering and Information Technology. His research interests include memory systems, processing-inmemory and machine learning hardware. Contact him at joao.sanchesferreira@safari.ethz.ch. 